\title{
INCORPORAÇÃO DAS ARMADURAS NO PROJETO DE ESTRUTURAS EM BIM
}

\author{
João Pedro Silva ${ }^{(1)(2)}$, Luís Costa Neves ${ }^{(1)}$, José Carlos Lino ${ }^{(2)}$ \\ (1) Universidade de Coimbra, Coimbra \\ (2) Newton - Consultores de Engenharia, Porto
}

\begin{abstract}
Resumo
No presente artigo abordam-se e comparam-se várias estratégias para modelação explícita de armaduras para betão armado em ambiente BIM. De facto, a complexidade geométrica inerente a este processo tem condicionado a generalização da modelação BIM de armaduras em contexto de projeto de estruturas de betão.

Este trabalho foi desenvolvido com recurso a Autodesk Revit, Autodesk Robot Structural Analysis, recorrendo-se também a extensões de apoio à modelação de armaduras como o Sofistik Reinforcement Detailing e o Naviate Rebar Extension. Também foram utilizadas extensões que permitem realizar a análise estrutural através do modelo analítico subjacente ao modelo BIM da geometria real, analisando-se a viabilidade de processos integrados de cálculo e modelação das armaduras. Foi também explorada a possibilidade de introdução de automatismos e melhorias à modelação de armaduras com base em scripts de linguagem de programação visual (Dynamo).

Todo o trabalho desenvolvido teve inicialmente como base dois casos de estudo relativamente simples: uma laje de geometria retangular apoiada em quatro pilares e uma laje de geometria não retangular, com uma abertura, apoiada em pilares e paredes. O artigo inclui discussão crítica e comparativa das metodologias adotadas.
\end{abstract}

\section{Introdução}

Em edições anteriores do Congresso Português de Building Information Modelling, já se podem encontrar artigos com estudos acerca da modelação de armaduras em ambiente BIM. Em Automatização da modelação BIM de armaduras no projeto de estruturas [1] podemos constatar que remete para a criação de um código que automatiza o processo de criação de armaduras em lajes bidirecionais; em Interoperabilidade BIM em projeto de estruturas de betão armado [2], evidencia-se a interoperabilidade entre os vários softwares utilizados, bem como a modelação de armaduras de modo a perceber quais as principais soluções na sua realização; e em Utilização de ferramentas BIM na pormenorização de armaduras de betão armado [3], destaca-se a importância da pormenorização e contabilização detalhada de armaduras, implicando a criação de Bar Schedules e Rebar Shapes. 
Estes artigos, apresentam um incremento de informação no processo de criação de modelos BIM, mais especificamente no que diz respeito à fase de modelação de armaduras. O estudo alvo deste artigo, incide também numa automatização desse processo, mas dando ênfase à exportação do modelo de armaduras que o Autodesk Robot Structural Analysis permite criar para o Autodesk Revit, tentando encontrar uma solução mais otimizada e o mais automática possível recorrendo às potencialidades do Dynamo (software de programação visual). Para além disso, esta investigação dedica-se também à exploração de uma extensão (Sofistik Analysis) que permite realizar uma análise estrutural no Autodesk Revit (Menu Structures), comparando esses resultados com os obtidos através do Autodesk Robot Structural Analysis. Posto isto, numa primeira fase, com o Autodesk Robot Structural Analysis será então realizada uma análise e respetivo dimensionamento estrutural que servirá de base para a modelação das armaduras no Autodesk Revit de forma a identificar e avaliar falhas existentes nos softwares. Após esta avaliação e identificação das limitações dos softwares, como alternativa à modelação das armaduras no Autodesk Revit, tendo em conta o vasto tempo que os modelos levam a ser desenvolvidos, é criado um modelo 3D automático para cada elemento estrutural no Autodesk Robot Structural Analysis e exportado para o software de modelação, de forma a otimizar o processo e criando um modelo de armaduras o mais automático possível.

Com a exportação das armaduras, será utilizado um software de programação visual (Dynamo), que permite criar novas funções no Autodesk Revit, servindo de apoio a essa transferência do modelo, corrigindo eventuais falhas que possam existir e otimizando o processo de criação do modelo 3D.

Na figura 1 apresenta-se um esquema do software utilizado neste trabalho.

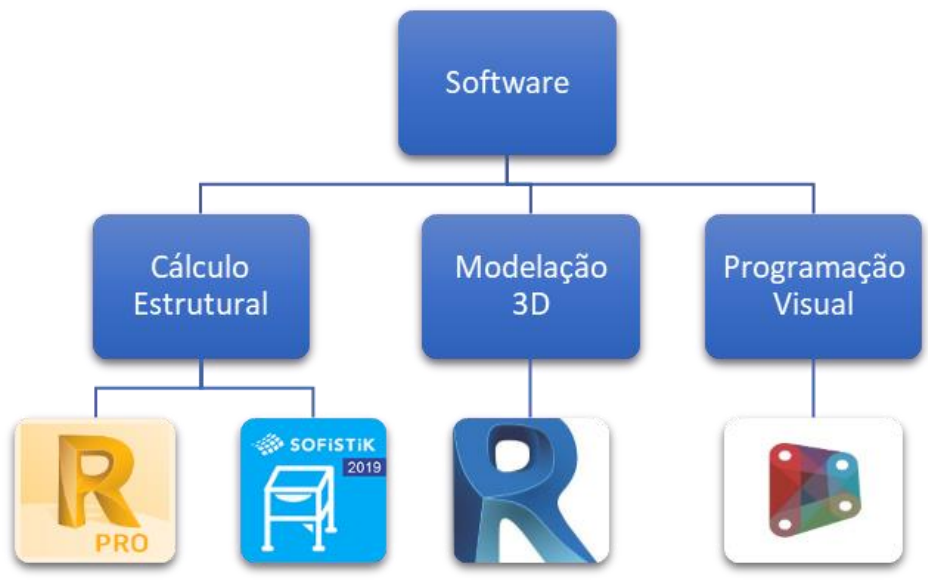

Figura 1: Esquema do software utilizado.

\section{Cálculo estrutural e modelos 3D}

Neste trabalho optou-se por utilizar o Autodesk Robot Structural Analysis para a análise estrutural de dois casos de estudo que podemos ver nas figuras 2 e 3, e o Autodesk Revit para criar o modelo 3D da estrutura e das respetivas armaduras, sendo que ambos os softwares pertencem à Autodesk, havendo desta forma uma boa interoperabilidade, facilitando a partilha de informação entre eles. 


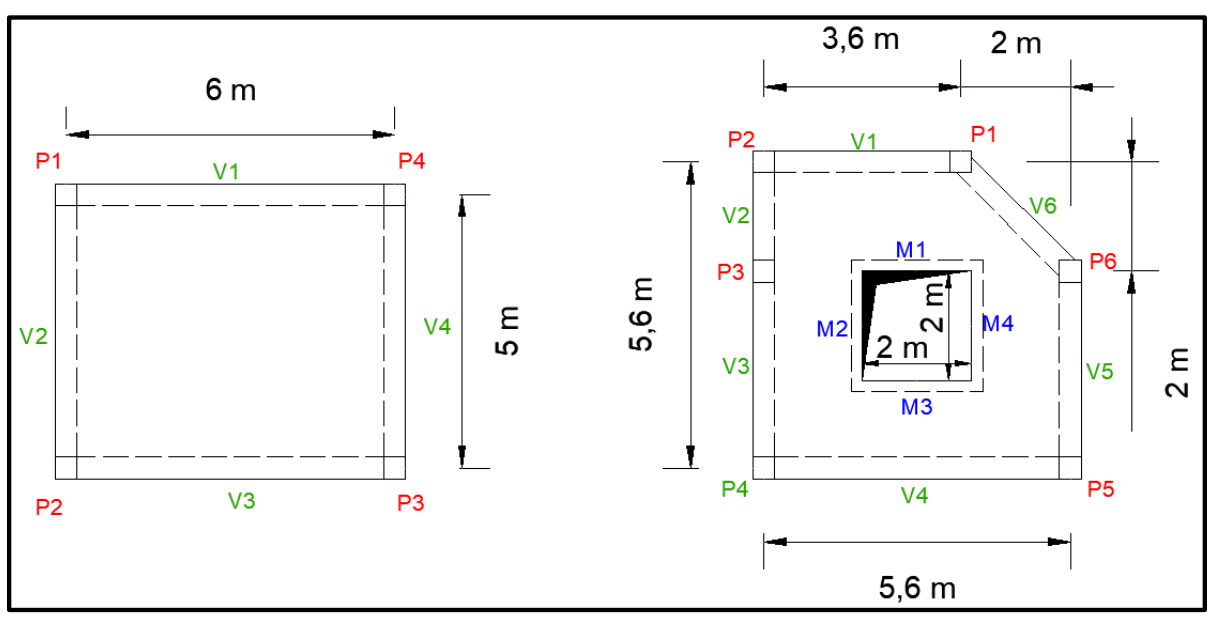

Figura 2: Dimensões em planta dos dois casos de estudo considerados.

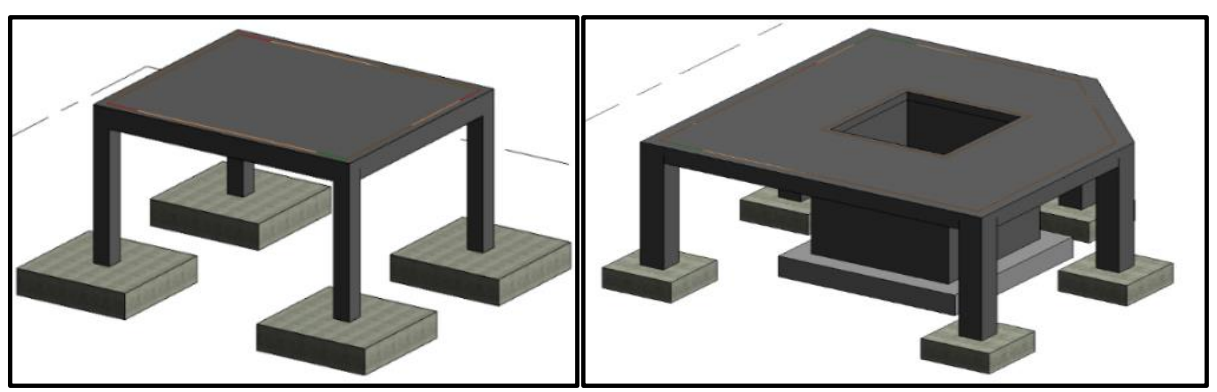

Figura 3: Modelos em Revit das estruturas 1 (esquerda) e 2 (direita).

Para além dos dois softwares da Autodesk, também surgiu a oportunidade de testar um outro software, o Sofistik Analysis, que permite realizar a análise estrutural diretamente no Autodesk Revit, sendo os resultados obtidos comparados posteriormente com os resultados obtidos pelo Robot, de forma a perceber se esta ferramenta dá uma resposta mais eficiente às solicitações do vasto mercado de software de cálculo, evitando a necessidade de recorrer a dois softwares diferentes para realizar o cálculo e a modelação 3D de armaduras.

\subsection{Análise estrutural (Robot vs Sofistik Analysis)}

Em primeiro lugar foram definidas apenas algumas combinações de ações de forma a poder calcular os esforços que servem de base para o dimensionamento. Importa realçar aqui que o objetivo desta pesquisa não é o de realizar uma análise estrutural ao pormenor, mas sim a exploração dos softwares de forma a otimizar a criação de um modelo 3D de armaduras.

De seguida, procedeu-se à análise estrutural no Autodesk Robot Structural Analysis e no Sofistik Analysis através dos modelos analíticos da figura 4 e 5 e comparação dos resultados nos dois softwares. 


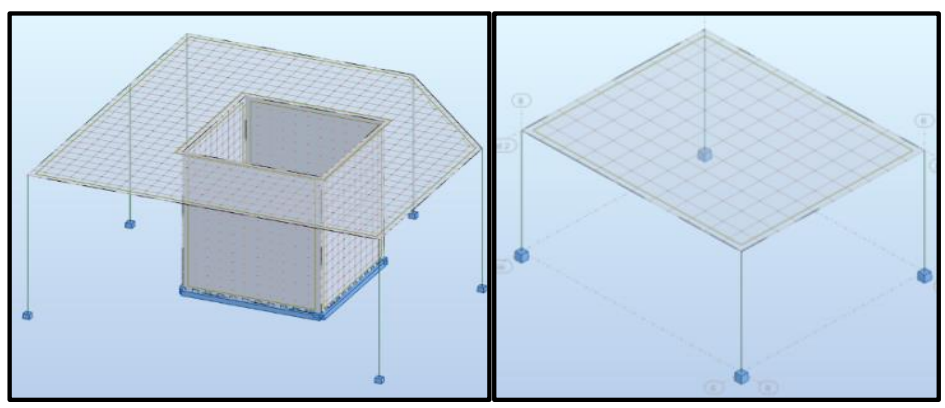

Figura 4: Modelos analíticos no Autodesk Robot Structural Analysis.

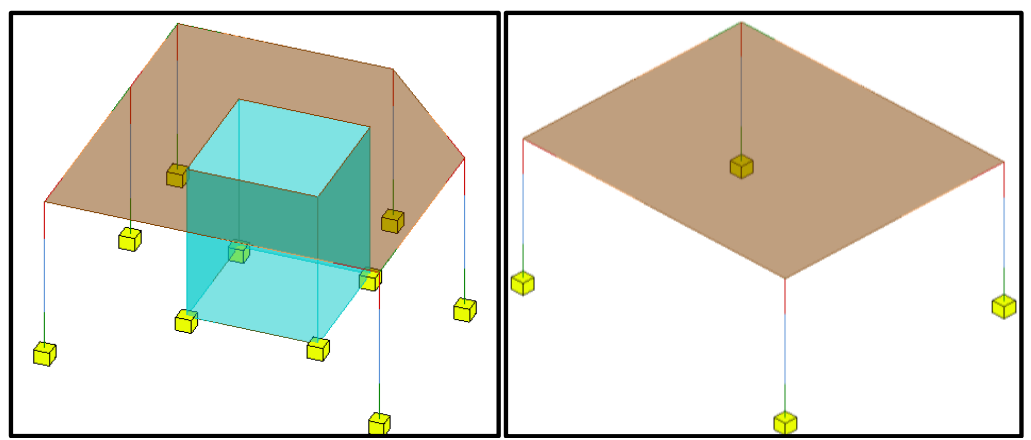

Figura 5: Modelos analíticos no Sofistik Analysis.

Como podemos observar nos mapas de momentos para lajes e paredes estruturais da figura 6, existem algumas ligeiras diferenças nos esforços que levantam algumas questões. Uma diferença no modelo do Sofistik é que não conseguimos visualizar automaticamente a divisão da malha de elementos finitos como no Autodesk Robot Structural Analysis, não sendo por isso tão simples verificar a malha gerada para a análise estrutural.

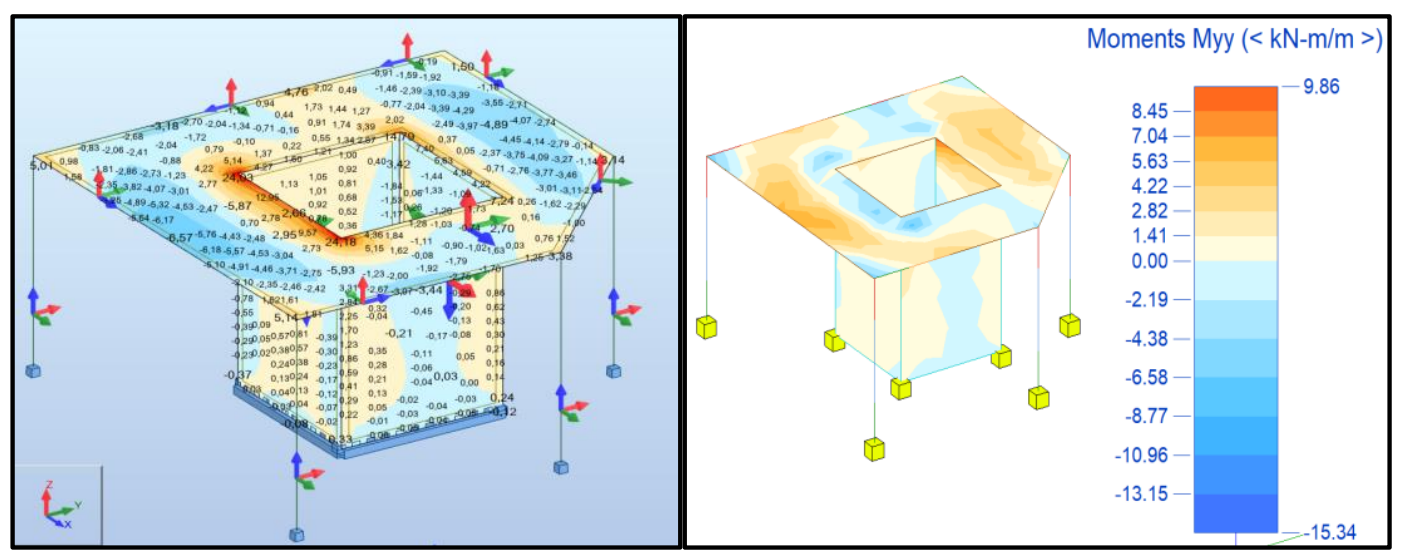

Figura 6: Mapas de momentos em lajes e paredes estruturais do caso de estudo 2 para a direção y entre o Robot (esquerda) e o Sofistik Analysis (direita).

Para além dos resultados obtidos para os momentos da laje e paredes estruturais, consultaramse os resultados para os restantes elementos da estrutura (vigas e pilares) como podemos ver nas figuras 7 e 8, constatando que os mesmos apresentam diferenças mínimas, levando a concluir que neste caso os resultados convergem para ambos modelos. 
Foram realizados os cálculos novamente, alterando os módulos de elasticidade nos dois softwares para verificar se os resultados obtidos seriam da mesma natureza, o que acabou por se verificar.

Para o Sofistik Analysis, e convergindo os resultados obtidos nos elementos do tipo viga e pilar, podemos dizer que o software pode vir constituir uma alternativa de mercado aos softwares de cálculo mais usados em Portugal, embora o caso do mapa de momentos que se verificou neste estudo deva ser analisado com mais pormenor para verificar a origem de ligeiras diferenças encontradas nos diagramas provenientes de cada um dos softwares.

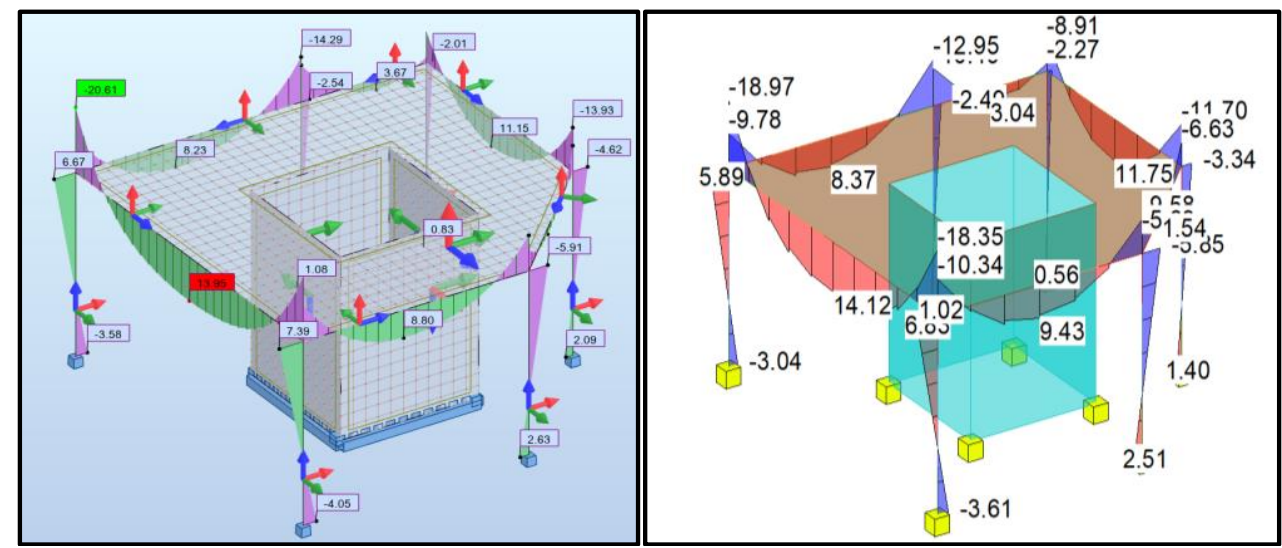

Figura 7: Momentos fletores segundo a direção y em ambos os softwares Robot (esquerda) e Sofistik Analysis (direita).

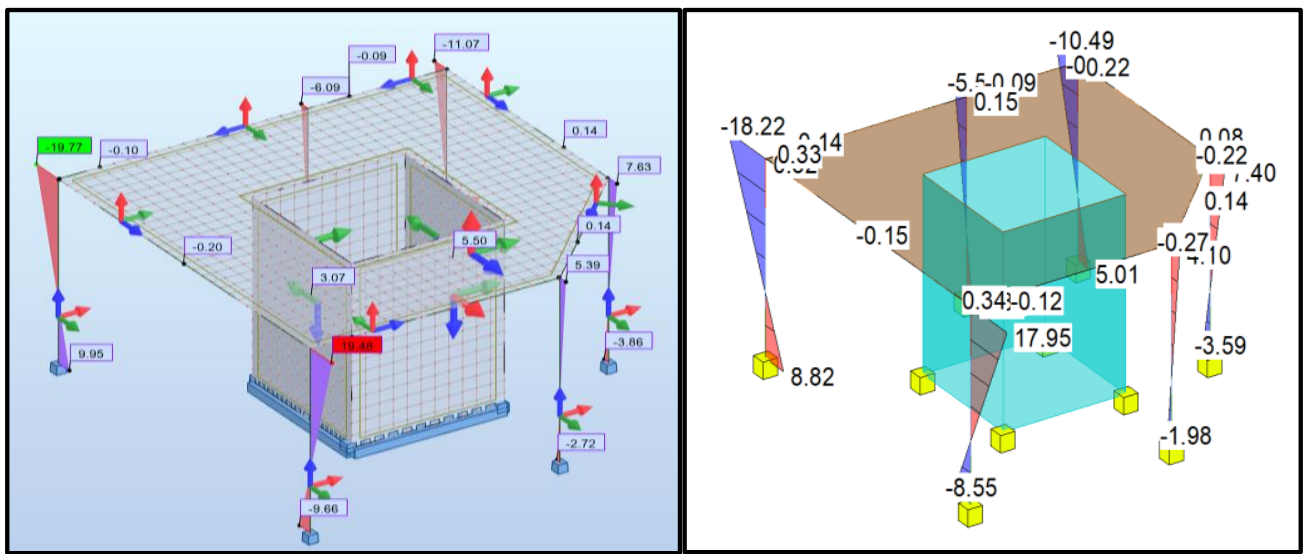

Figura 8: Momentos fletores segundo a direção z em ambos os softwares Robot (esquerda) e Sofistik Analysis (direita).

\subsection{Modelos 3D}

Após a análise estrutural, realizou-se o dimensionamento das estruturas com base nos resultados obtidos pelo Autodesk Robot Structural Analysis e procedeu-se à modelação das armaduras no Autodesk Revit, de forma a avaliar as limitações do software e encontrar novas alternativas a este tipo de modelação que otimizem o processo de produção do modelo (ver figura 9). 


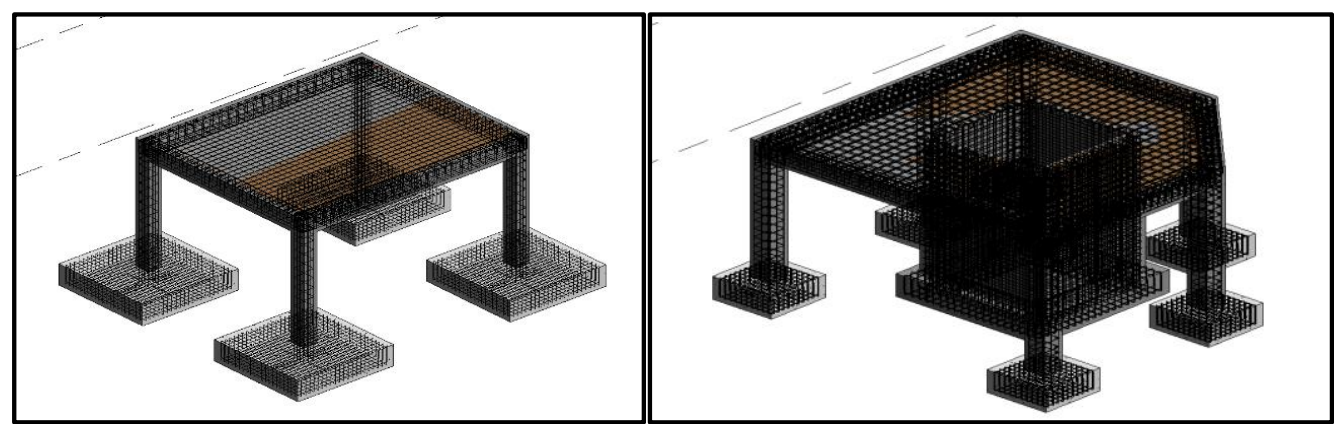

Figura 9: Modelos de armaduras no Autodesk Revit.

Na modelação das armaduras, encontraram-se diversas dificuldades, sobretudo nas armaduras de lajes e paredes e nas ligações entre os elementos estruturais onde ocorrem várias vezes sobreposições de varões. Para contornar alguns dos inconvenientes encontrados, foram adicionadas duas extensões ao Revit que apresentam algumas funções não presentes neste software e que ajudaram na conceção do modelo de armaduras. Foram elas o Sofistik Reinforcement e o Naviate Rebar Extension. Mesmo com o apoio destas extensões de modelação, continuou a haver limitações que dificultaram a obtenção do modelo de armaduras final pretendido, verificando-se ainda alguma morosidade no processo.

Neste sentido, através das conclusões tiradas nestes dois modelos, o objetivo focou-se em tentar encontrar uma solução alternativa que permitisse contornar estas limitações e tornar este processo o mais automatizado possível.

\section{Automatização Robot vs Revit}

Uma das formas de tornar este processo o mais automático possível, passa por evitar a modelação da totalidade da armadura no Autodesk Revit, procurando uma solução que permita automatizar a criação do modelo 3D de armaduras.

O Autodesk Robot Structural Analysis, para além da análise estrutural, também permite fazer o dimensionamento das armaduras para cada elemento estrutural com base nessa mesma análise estrutural, consoante as combinações de ações e as características dos materiais, criando desta forma, automaticamente, um modelo 3D das armaduras (ver figura 10).

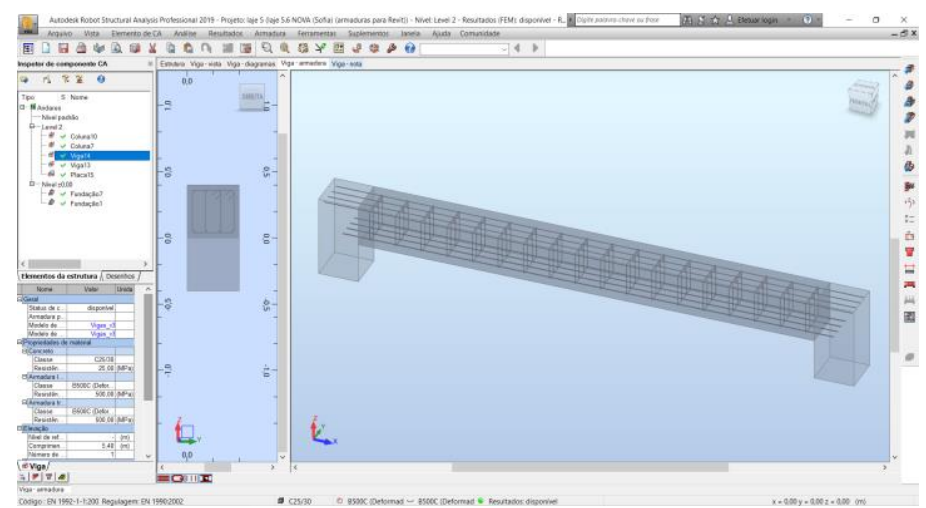

Figura 10: Exemplo do modelo 3D da armadura de uma viga calculada através do Autodesk Robot Structural Analysis. 
Dado que estes dois softwares pertencem à Autodesk, apresentando uma boa interoperabilidade entre si, é possível exportar o modelo de armaduras do software de análise e dimensionamento estrutural para o Autodesk Revit. Desta forma a conceção do modelo tridimensional de armaduras será mais automatizada e levará menos tempo a ser concluída.

O Autodesk Robot Structural Analysis, para além das armaduras em vigas, também permite fazer o dimensionamento dos restantes elementos estruturais como pilares, lajes ou sapatas. No entanto, só foi possível exportar os elementos do tipo viga e pilar para o Autodesk Revit.

\subsection{Exportação do modelo}

Após a exportação do modelo, verificou-se para o caso específico das vigas que a armadura transversal não é exportada corretamente, apresentando anomalias como podemos ver na figura 11. Neste caso houve um deslocamento da armadura para uma das extremidades da viga.

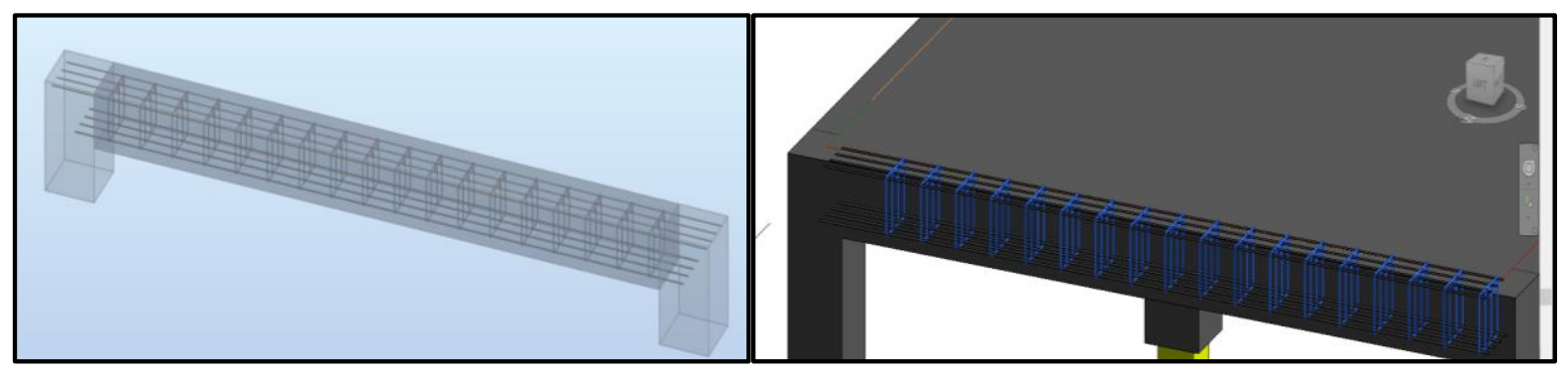

Figura 11: Modelo de armaduras no Autodesk Robot Structural Analysis (esquerda) e no Autodesk Revit (direita).

Após este resultado, realizou-se o processo de exportação das armaduras múltiplas vezes com ambas as estruturas, tentando encontrar a origem do problema, o qual persistiu em todos os elementos do tipo viga. Por vezes, também a armadura longitudinal não ficou localizada no local pretendido. Através deste resultado, e não se conseguindo identificar a origem concreta do problema, optou-se por procurar uma alternativa para corrigir estas questões.

\subsection{Dynamo}

Para contornar as incompatibilidades encontradas na exportação das armaduras e tornar o processo de modelação das mesmas o mais automático possível, decidiu-se recorrer ao Dynamo, que é um software de programação visual que trabalha como plug-in no Autodesk Revit, permitindo criar novas funções ou melhorias na modelação. Decidiu-se então, através deste software, criar uma aplicação que corrija os problemas encontrados após a exportação das armaduras e que ajude nesta fase do projeto estrutural a otimizar a produção do modelo 3D de armaduras.

Neste sentido, foi desenvolvido um código (plug-in 1) em Dynamo onde o objetivo seria alterar as coordenadas do centro geométrico do conjunto de estribos, e sobrepor as mesmas com as coordenadas do centro geométrico da viga (ver figura 12). No entanto, ainda que este fosse o caminho mais lógico a seguir, foi encontrado um erro quando se correu o programa numa fase final do código que não permitiu definir a posição final das armaduras (ver figura 13). 


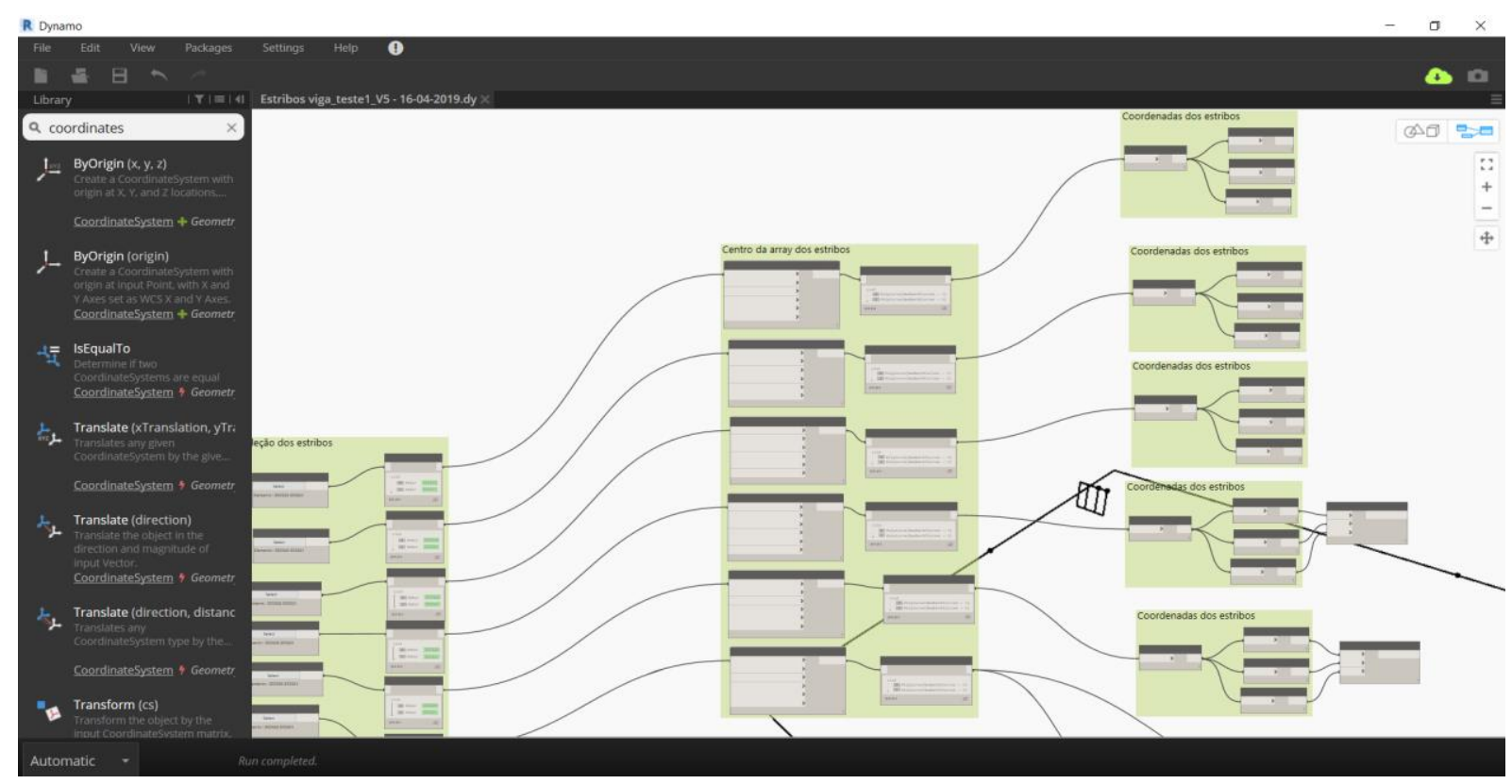

Figura 12: Plug-in 1 desenvolvido em Dynamo.

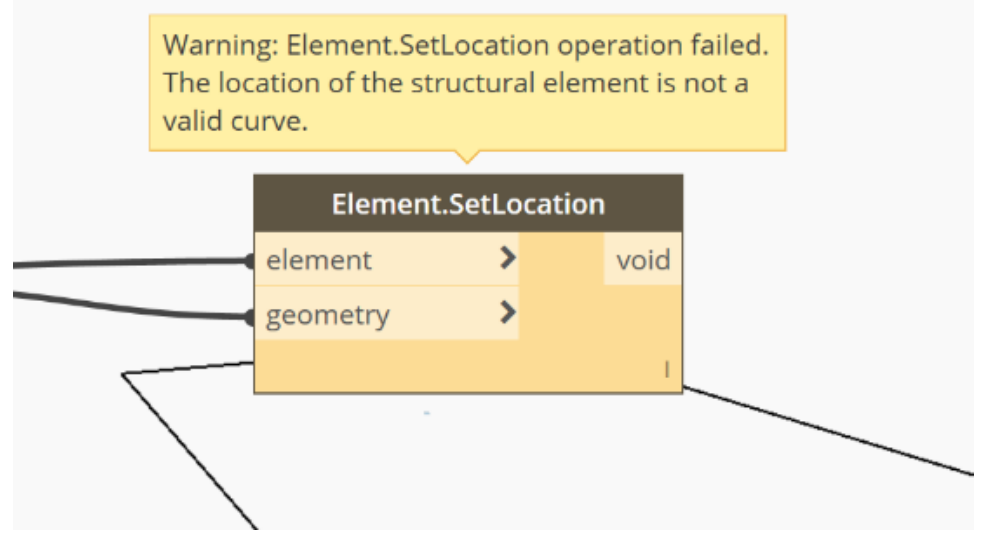

Figura 13: Erro ocorrido na definição da posição final dos estribos.

Após algumas tentativas de corrigir o código, sem sucesso, decidiu-se seguir outro caminho, criando um novo código (plug-in 2) que necessita de alguns inputs por parte do utilizador, nomeadamente a seleção da armadura ou outro objeto que se pretenda alterar e a introdução da distância pretendida em qualquer direção cartesiana (x, y, z) (ver figura 14).

Para além deste caso específico onde há a necessidade de corrigir os estribos dos elementos estruturais do tipo viga, este plug-in também poderá ser utilizado para alterar outro tipo de armadura, ou até mesmo elementos da estrutura, de forma mais rápida e automática.

Na figura 15 apresenta-se um esquema da execução do programa criado em Dynamo (plug-in 2). 


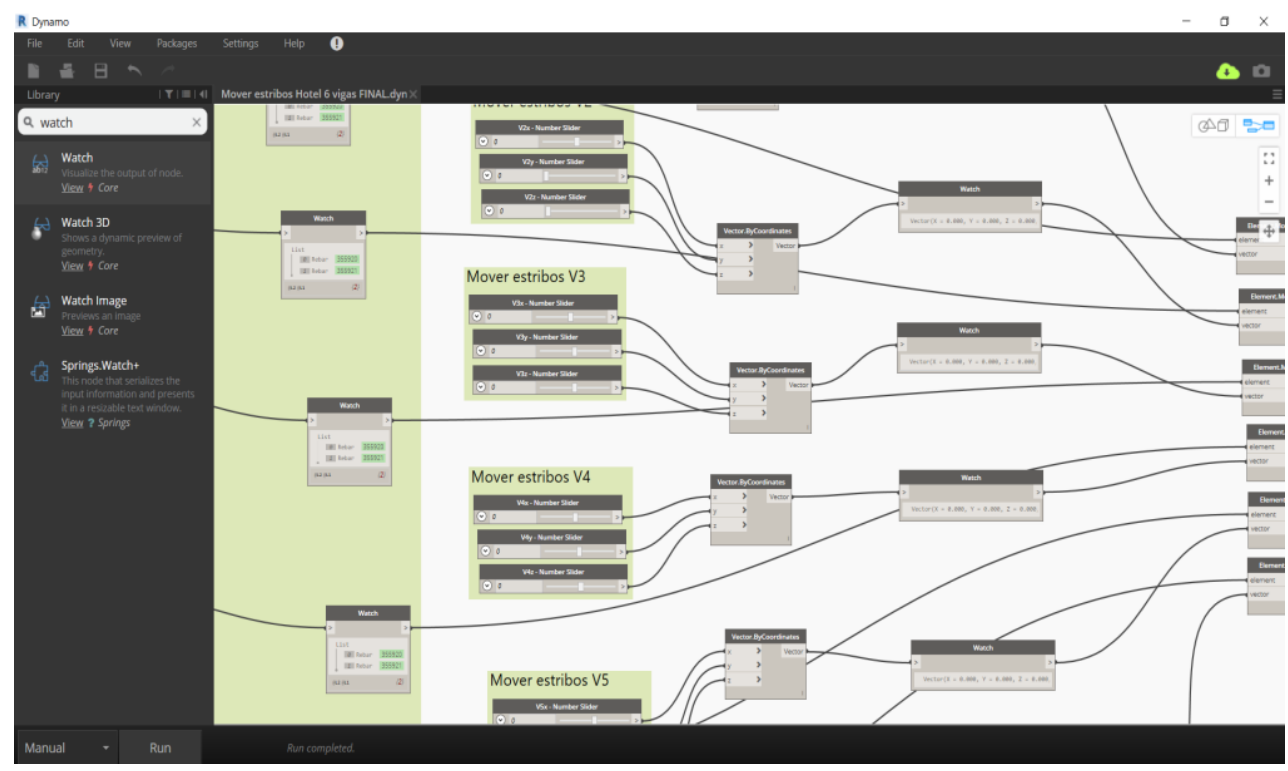

Figura 14: Plug-in 2 desenvolvido em Dynamo.
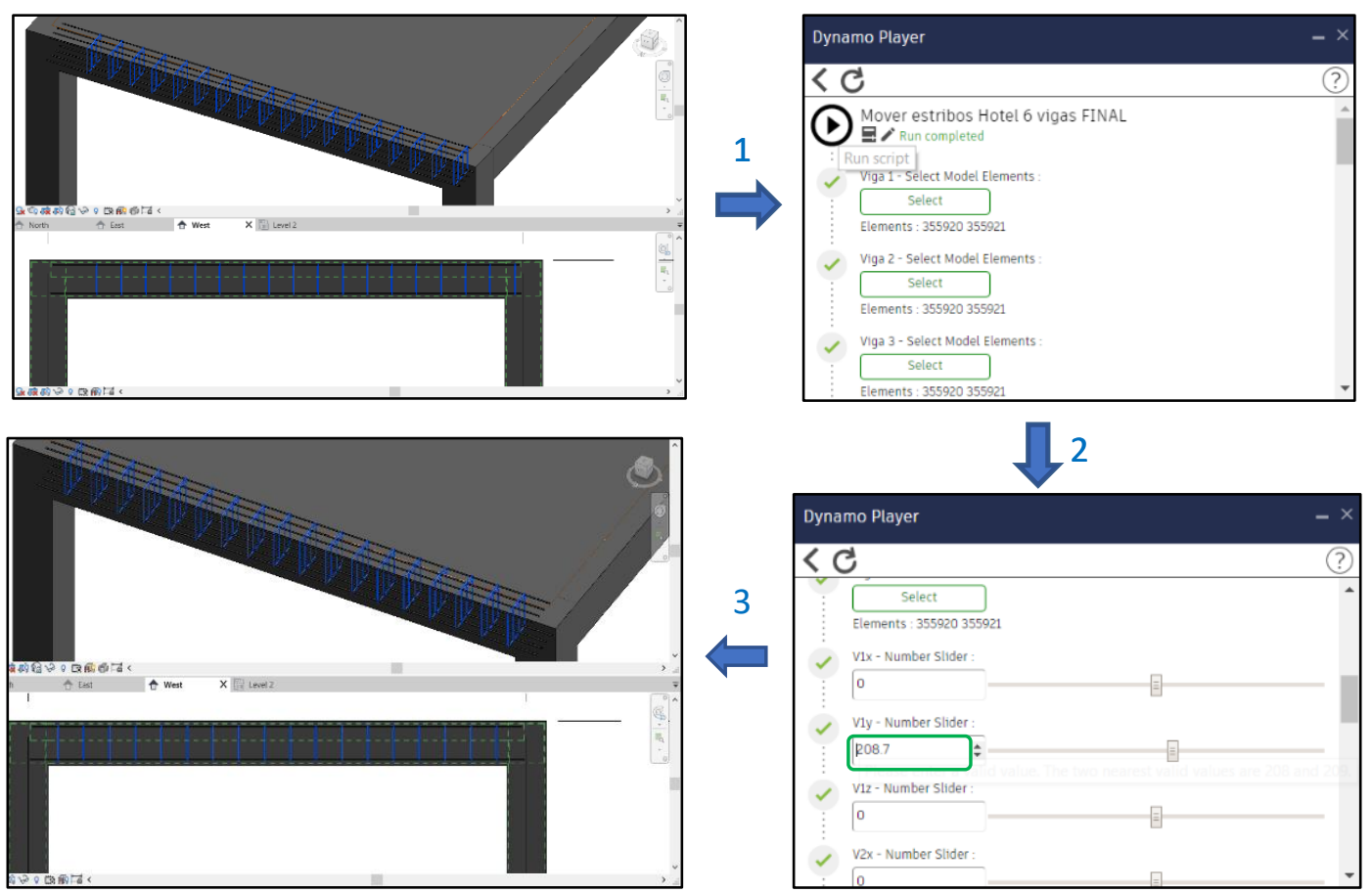

Figura 15: Exemplo da execução do plug-in 2 numa das vigas da estrutura 1.

\section{Conclusão}

Neste trabalho, o objetivo principal focou-se na modelação em ambiente BIM de armaduras de betão armado, no sentido de avaliar as potencialidades do Autodesk Revit e do Robot Structural Analysis e encontrar novos métodos que otimizem esta fase do projeto de estruturas e automatizando o processo de criação do modelo 3D. 
Relativamente à fase de análise estrutural, foram comparados dois softwares baseados no método dos elementos finitos, nomeadamente o Autodesk Robot Structural Analysis e o Sofistik Analysis, onde se pôde comparar os momentos fletores em elementos do tipo laje e parede estrutural. Apesar de ligeiras diferenças entre os softwares em lajes, verificou-se uma semelhança entre os resultados obtidos para os momentos fletores dos restantes elementos estruturais de ambas as estruturas. Desta forma, pode concluir-se a eficácia de ambos os softwares na análise estrutural. O Sofistik é uma ferramenta que apresenta potencial para ter margem de progressão no mercado nacional, como otimização desta fase do projeto estrutural, evitando deste modo o uso de dois softwares para a análise, dimensionamento e produção do modelo 3D de armaduras.

No assunto da modelação de armaduras através do Autodesk Revit, pode concluir-se que apresenta limitações na conceção do modelo de armaduras, principalmente em lajes, paredes ou ligações entre elementos estruturais, tornando o processo algo moroso; tem vindo a apresentar uma grande margem de progressão sobre essas limitações; e permite criar novos comandos de apoio à modelação de armaduras através do Dynamo.

$\mathrm{Na}$ exportação do modelo de armaduras do Autodesk Robot Structural Analysis para o Autodesk Revit verificaram-se algumas dificuldades nessa exportação, principalmente na posição das armaduras transversais de elementos do tipo viga. Neste sentido, recorrendo à programação visual em Dynamo em que foi desenvolvido um código que corrigiu o problema encontrado, otimizando assim a conceção do modelo 3D, aproveitando assim parte da modelação das armaduras no Autodesk Revit.

O código desenvolvido, para além de ser aplicado a elementos do tipo viga, pode ser adaptado para diferentes tipos de armadura e de modelações qualquer estruturais.

\section{Referências}

[1] H. Pires, J. Lino e C. Rodrigues, "Automatização da modelação BIM de armaduras no projeto de estruturas", $2^{\circ}$ Congresso Português de Building Information Modelling - Porto, Portugal, 2018

[2] J. Alves, J. Lino e L. C. Neves, "Interoperabilidade BIM em projeto de estruturas de betão armado", $2^{\circ}$ Congresso Português de Building Information Modelling - Porto, Portugal, 2018

[3] J. Lima, J. Silva, V. Pascoal, A. Monteiro, "Utilização de ferramentas BIM na pormenorização de armaduras de betão armado", $2^{\circ}$ Congresso Português de Building Information Modelling - Porto, Portugal, 2018

[4] C. M. Eastman, P. Teicholz, R. Sacks, and K. Liston, BIM handbook : a guide to building information modeling for owners, managers, designers, engineers, and contractors. Hoboken, N.J.: Wiley, 2008.

[5] J. Silva (2019), "Incorporação das armaduras no projeto de estruturas em BIM". Dissertação submetida para satisfação dos requisitos do grau de mestre em engenharia civil, Faculdade de Ciências e Tecnologias da Universidade de Coimbra, Departamento de Engenharia Civil. 